\title{
Precession Electron Diffraction for SiC Grain Boundary Characterization in Unirradiated TRISO Fuel
}

\author{
T. M. Lillo ${ }^{a}$, I. J. van Rooyen ${ }^{b}$ and Y. Q. $W^{c, d}$ \\ ${ }^{a}$ Materials Science \& Engineering Department, Idaho National Laboratory, \\ Idaho Falls, ID 83415-2211, USA \\ Corresponding author. Tel +1 2085269746 \\ E-mail address: thomas.lillo@inl.gov \\ ${ }^{b}$ Fuel Performance and Design Department, Idaho National Laboratory, \\ Idaho Falls, ID 83415-6188, USA \\ 'Micron School of Materials Science and Engineering, Boise State University, \\ Boise, ID 83725-2090, USA \\ ${ }^{d}$ Center for Advanced Energy Studies, \\ Idaho Falls, ID 83401, USA
}

\begin{abstract}
Precession electron diffraction (PED), a transmission electron microscopy-based technique, has been evaluated for the suitability for evaluating grain boundary character in the SiC layer of tristructural isotropic (TRISO) fuel. This work reports the effect of transmission electron microscope (TEM) lamella thickness on the quality of data and establishes a baseline comparison to SiC grain boundary characteristics, in an unirradiated TRISO particle, determined previously using a conventional electron backscatter diffraction (EBSD) scanning electron microscope (SEM)-based technique. In general, it was determined that the lamella thickness produced using the standard focused ion beam (FIB) fabrication process ( $\sim 0 \mathrm{~nm}$ ), is sufficient to provide reliable PED measurements, although thicker lamellae $(\sim 120 \mathrm{~nm})$ were found to produce higher quality orientation data. Also, analysis of SiC grain boundary character from the TEM-based PED data showed a much lower fraction of low-angle grain boundaries compared to SEM-based EBSD data from the SiC layer of a TRISO-coated particle made using the same fabrication parameters and a SiC layer deposited at a slightly lower temperature from a surrogate TRISO particle. However, the fractions of high-angle and coincident site lattice (CSL)related grain boundaries determined by PED are similar to those found using SEM-based EBSD. Since the grain size of the SiC layer of TRSIO fuel can be as small as $250 \mathrm{~nm}$ [Kirchhofer, et al., 2013], depending on the fabrication parameters, and since grain boundary fission product precipitates in irradiated TRISO fuel can be nano-sized, the TEM-based PED orientation data collection method is preferred to determine an accurate representation of the relative fractions of low-angle, high-angle, and CSL-related grain boundaries. It was concluded that although the resolution of the PED data is
\end{abstract}


better by more than an order of magnitude, data acquisition times may be significantly longer or the number of areas analyzed needs to be significantly greater than the SEM-based method to obtain a statistically relevant distribution. Also, grain size could be accurately determined but significantly larger analysis areas would be required than those used in this study.

Key words: Precession electron diffraction, TRISO, SiC, grain boundary characteristics

\section{INTRODUCTION}

The SiC layer of tristructural isotropic (TRISO)-coated fuel acts as the primary barrier for the containment of fission products produced in the uranium-containing kernel. However, experience shows that a significant amount of certain fission products can be released through apparently intact SiC layers [van der Merwe, 2009; Nabielek, et al., 1977; Demkowicz, et al., 2012]. The diffusion rates of fission products, especially $\mathrm{Ag}$, through the $\mathrm{SiC}$ layer reported by researchers vary by orders of magnitude [Malherbe, 2013]. Also, a number of researchers conclude that lattice diffusion of $\mathrm{Ag}$ in $\mathrm{SiC}$ is negligible, even at high temperatures [Schrader, et al., 2011; Khalil, et al., 2011]. Therefore, many consider grain boundary diffusion to be the dominant release mechanism. Also, recent post irradiation examination results from the Advanced Gas Reactor (AGR)-1 experiment [Lillo and van Rooyen, 2015; van Rooyen, et al., 2014] and a modelling study [Rabone and López-Honorato, 2015] imply that grain boundary character may significantly influence the diffusion of fission products.

Only recently has it been possible to identify fission product precipitates on individual grain boundaries and, at least, qualitatively analyze their composition with scanning transmission electron microscopy (STEM)/transmission electron microscope (TEM) using z-contrast imaging and energy dispersive spectroscopy (EDS) [van Rooyen, et al., 2014]. However, in order to elucidate the influence of grain boundary character on fission product migration in individual grain boundaries, it is necessary to employ techniques that can determine the misorientation across individual grain boundaries that contain fission products as well as across grain boundaries that do not contain fission products. While scanning electron microscope (SEM)-based electron backscatter diffraction (EBSD) can determine grain boundary misorientation, it is not possible to identify nano-sized, fission product precipitates on the grain boundaries and accurately identify the individual fission product elements that are present in these precipitates. Methods to determine grain orientations, and subsequently grain boundaries misorientations, in the TEM based on Kikuchi bands are relatively slow; often, the Kikuchi bands are not sufficiently prominent to allow analysis of the orientation.

Recently, orientation identification techniques based on precession electron diffraction (PED) have advanced to the point where automated PED pattern collection and analysis systems are now commercially available [Rauch, et al, 2008]. PED allows the resulting diffraction pattern at a point to be matched with one in a PED pattern database consisting of simulated diffraction patterns generated over the range of possible orientations. The advantage of PED is that it utilizes a very small electron beam spot size ( $\sim \mathrm{nm}$ or less) and the interaction volume is on the order of the beam size since the sample is very thin. Both enable a very small step size and high spatial resolution, allowing orientation analysis at the nano-level. Therefore, it would appear that PED techniques are well-suited for analysis of the nano-sized grain boundary fission product precipitates.

This work reports on the effect of sample thickness on the quality of PED data as well as the grain boundary character and coincident site lattice (CSL)-related grain boundary distributions in the SiC layer of unirradiated TRISO fuel using PED-based techniques in the TEM. These results are compared to the results of SEM-based EBSD characterization studies found in literature. The goal is to evaluate 
the suitability of the PED technique for evaluating grain boundary character as well as characterizing the grain boundary character distribution prior to exposure to neutron irradiation.

\section{EXPERIMENTAL}

An unirradiated TRISO particle was selected for analysis of the SiC layer. The TRISO particle was made using Variant 3 fabrication parameters. Generally, the SiC layer in Variant 3 TRISO fuel particles was deposited at a lower temperature $\left(\sim 1425^{\circ} \mathrm{C}\right)$ than in baseline TRISO fuel particles $\left(\sim 1500^{\circ} \mathrm{C}\right)$ and a mixture of $\mathrm{Ar}+\mathrm{H}_{2}$ gas was used as the fluidizing gas versus pure $\mathrm{H}_{2}$ for the baseline TRISO fuel particles. Details concerning the rationale of different variants and the actual fabrication parameters can be found in Refs. [Kirchhofer,et al., 2013; Maki, 2009; Hunn, et al., 2006]. The particle was mounted in epoxy, ground to approximately the mid-plane of the particle, and polished. Figure 1 shows the polished cross section and the locations of two TEM samples, referred to as "Inner" and "Outer" samples, which were prepared using standard focused ion beam (FIB) techniques. The plane of these samples is parallel to the radial growth direction of the SiC layer and capture the columnar nature of the SiC grains. A final nominal thickness of the Inner sample was targeted at $50 \mathrm{~nm}$, while the final nominal thickness of the Outer sample was targeted at $150 \mathrm{~nm}$ to explore the effect of sample thickness on the quality of the PED orientation data.

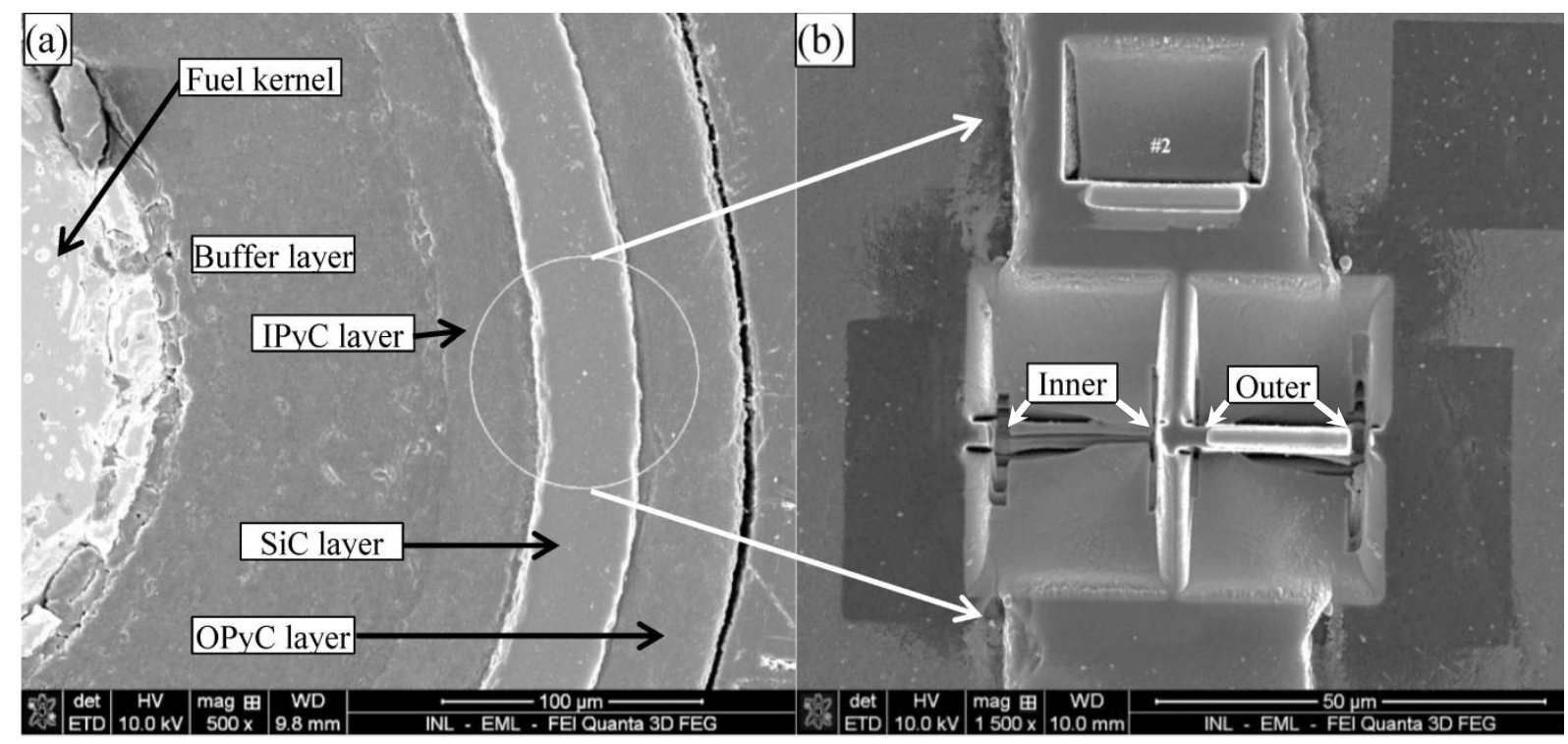

Figure 1. Secondary electron image of (a) the polished cross section of the TRISO-coated particle (Variant 3), and (b) the locations of FIB TEM samples taken from the inner and outer regions of the $\mathrm{SiC}$ layer.(IPyC = Inner Pyrolytic Carbon layer, OPyC = Outer Pyrolytic Carbon layer). The extent of the Inner and Outer samples is defined by the arrows in (b). The Inner sample has already been removed in (b).

The crystallographic orientations of the grains in two areas on the Inner sample and three areas on the Outer sample were analyzed using PED with the ASTAR system (NanoMegas, Inc.) on a Tecnai TF30-FEG STwin, operating at $300 \mathrm{kV}$, at the Center for Advanced Energy Studies. The spot size during data collection was measured to be approximately $5 \mathrm{~nm}$ while the step size was $10.4 \mathrm{~nm}$. Crystallographic information from areas on the order of $2 \mu \mathrm{m} \times 2 \mu \mathrm{m}$ was collected, resulting in approximately 40,000 points in each scan. The crystallographic orientation, as determined by the ASTAR analysis software, is reported by the manufacturer to be within approximately 1 degree of the actual orientation. 
The crystallographic information determined with the ASTAR software was imported into Orientation Imaging Microscopy (OIM) analysis software (EDAX OIM v7.1.0), which was used to determine the PED pattern quality, the average SiC grain size and the grain boundary character distribution in each analysis area. Clean up of the data was kept to a minimum. Initially, a dilatation operation was performed using a grain tolerance angle of 2 degrees and minimum grain size of 5 pixels (approximately equal to features with a 30-nm equivalent diameter). This was followed by neighbor orientation correlation operation using a grain tolerance angle of 2 degrees, a minimum confidence index of 0 , and a cleanup level of 1 . Generally, less than $3 \%$ of the points in a scanned area were affected by the cleanup procedures. Grains were defined as those features exceeding approximately $45 \mathrm{~nm}$ in diameter as well as having a misorientation angle, $\theta$, greater than or equal to 2 degrees (which is approximately the angular resolution during data collection).

\section{RESULTS}

\subsection{Suitability of PED for Crystallographic Orientation Determination in the SIC Layer}

Automated precession diffraction offers the potential to quickly map crystallographic information with nanometer resolution on TEM samples [Rauch, et al, 2008]. The ASTAR system collects PED patterns and yields orientation information point-by-point across a scanned area. The crystallographic orientation is then determined by matching the PED pattern to a simulated pattern in an automated routine. The accuracy of pattern matching may potentially be affected by the thickness of the sample. Areas that are sufficiently thin may not produce strong diffraction spots, which are used in the pattern matching routine. Overly thick areas (those on the order of the grain size or larger) may have a high frequency of overlapping grains illuminated by the electron beam during PED pattern collection. This would result in overlapping diffraction patterns and would complicate or reduce the accuracy of the pattern-matching routine. Therefore, the appropriateness of the sample thickness used in this work was evaluated. The thickness of five areas analyzed on these two samples (two on the Inner sample and three on the Outer sample) was determined by the electron energy loss spectrometry (EELS) method (e.g., location and height of the plasmon peak). Three areas were found to be approximately $80 \mathrm{~nm}$ thick, while two others were found to be approximately $120 \mathrm{~nm}$ thick. The reliability parameter calculated by the ASTAR software was taken as the primary indication of the data quality with higher values of the reliability parameter, indicating a higher confidence in the crystallographic orientation assigned by the ASTAR software. The distribution of the reliability parameter for each PED pattern in the five analysis areas is shown in Figure 2. The frequency distributions of the thicker areas are centered on higher values of the reliability parameter compared to the thinner regions. From this it appears that thicker samples generally produce higher quality orientation data than thinner samples. However, the reliability parameter is sufficiently high at both 80 and $120 \mathrm{~nm}$ so that inaccuracies in the automated indexing routine in the ASTAR software are virtually negligible.

It should be noted that at some critical thickness, grains will overlap significantly. This results in the degradation of the orientation data and more extensive zones of orientation uncertainty between grains. This critical thickness will likely be grain-size dependent with the critical thickness decreasing with decreasing grain size. However, the FIB-prepared samples from the SiC layer of this TRISOcoated fuel appear to be of sufficient thickness to produce high-quality orientation data and yet not excessively thick. 


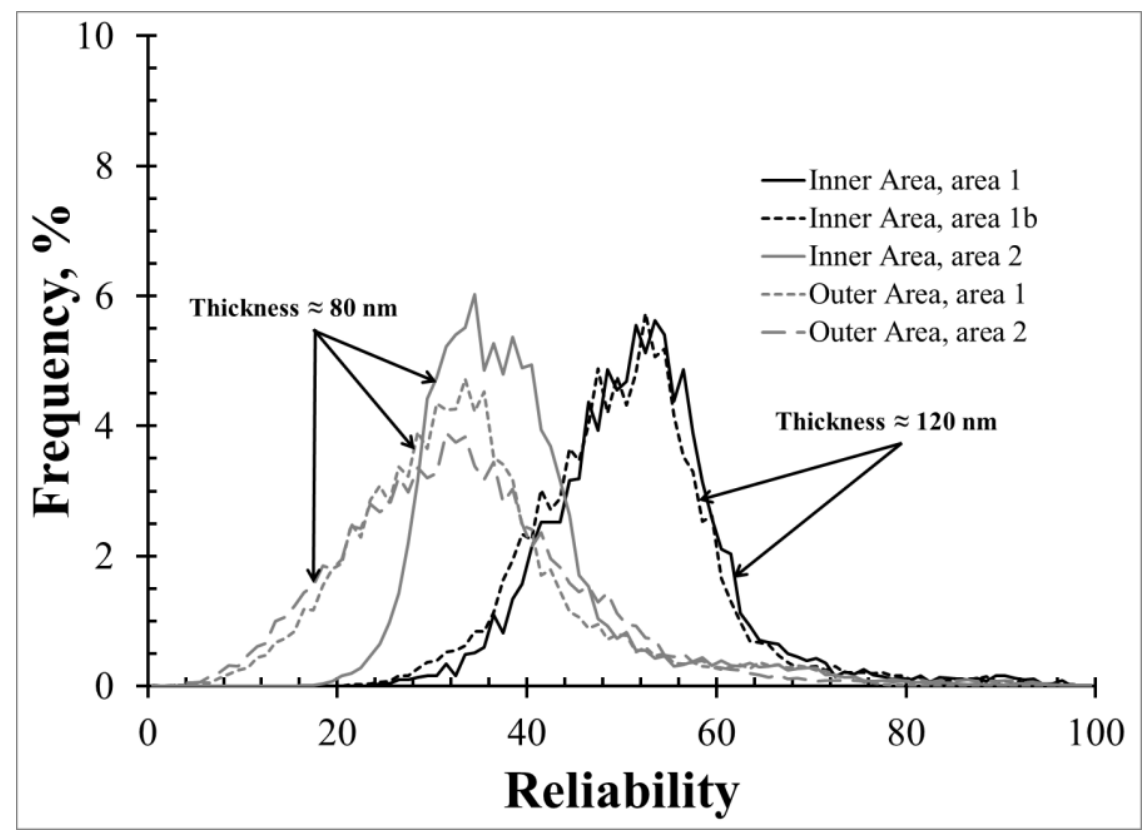

Figure 2. Frequency distribution of the reliability parameter showing thicker samples yield higher quality orientation data.

\subsection{Grain Size}

Figure 3 shows the TEM image of an analyzed area in the Outer sample along with the resulting orientation image. The microstructure consists of a few relatively large grains with interspersed fine grains. High-angle grain boundaries $\left(\theta \geq 15^{\circ}\right)$ are outlined in black, while low-angle grain boundaries $\left(2^{\circ} \leq \theta<15^{\circ}\right)$ are in white. The majority of the grain boundaries in this figure are high angle.

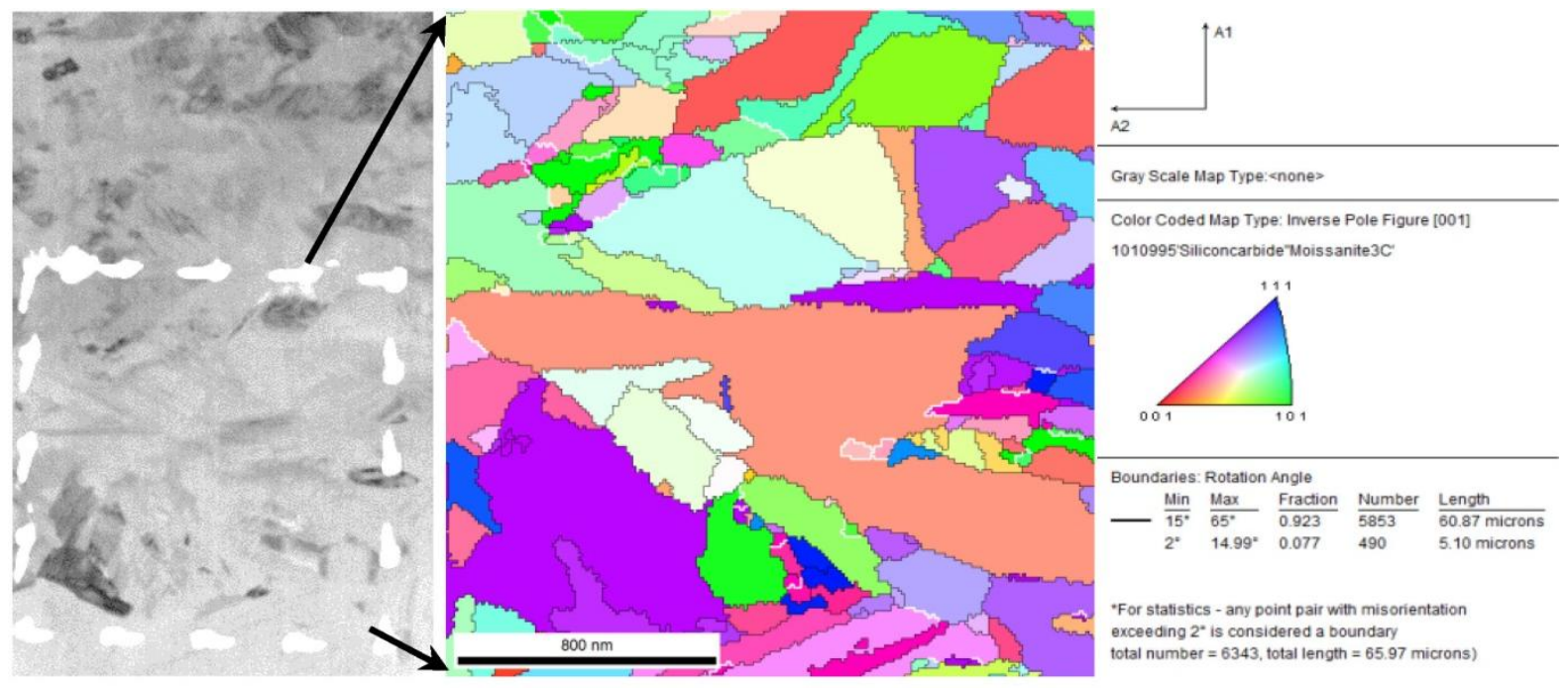

Figure 3. A TEM image of an area of analysis (outlined in white) on the Outer sample (left) with the corresponding orientation map (middle) and color legend (right). High-angle grain boundaries are in black while the low-angle boundaries are in white.

Once the orientation data generated by the ASTAR program is imported into the OIM analysis program, all the typical grain and grain boundary information is available. Figure 4 shows the grain size distribution determined for the Inner and Outer samples of the SiC layer. Each distribution includes the combined data from all the areas scanned on each sample. The variation in the grain size 
distribution for each scanned area was used in the calculation of the standard error, which is shown as the error bars in Figure 4. The grain size distributions do not differ significantly between the Inner and Outer samples of the SiC layer. Additionally, the average grain size calculated from these distributions, and shown in Figure 4, are not statistically different, regardless of whether a number average or an area-weight average is compared.

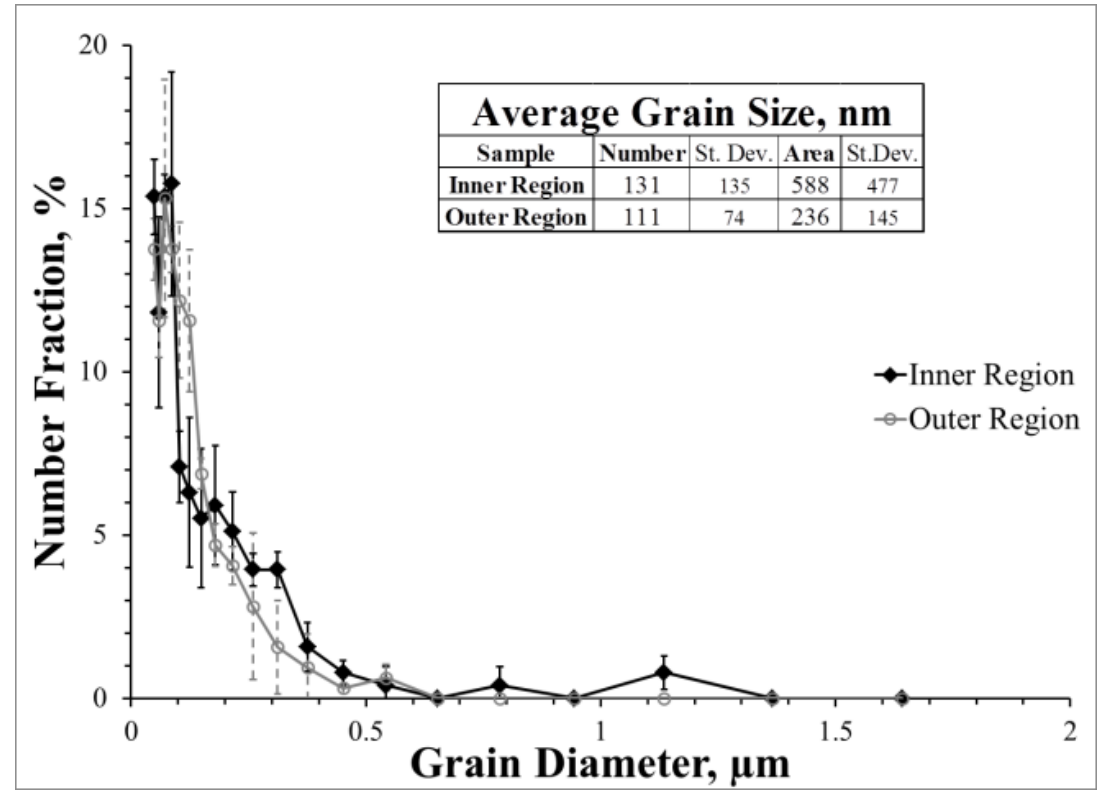

Figure 4. Comparison of the grain size distributions in the Inner and Outer samples.

\subsection{Grain Boundary Distributions}

The orientation data was also used to calculate the grain boundary character distribution. Grain boundaries were categorized as either low angle $\left(2^{\circ} \leq \theta<15^{\circ}\right), C S L$ - related $(\Sigma 3-\Sigma 29)$ or high angle $(\theta$ $\left.\geq 15^{\circ}\right)$. The high-angle grain boundary fraction did not include those grain boundaries identified as CSL-related grain boundaries. Figure 5 shows that most grain boundaries are CSL-related grain boundaries. The remaining grain boundaries are predominantly random high-angle, while only a small fraction is considered to be low angle. The trends exhibited by the Inner and Outer samples are similar and not statistically different (i.e., the grain boundary character distribution is the same in both inner as well as outer areas of the SiC layer in this Variant 3, TRISO-coated fuel particle).

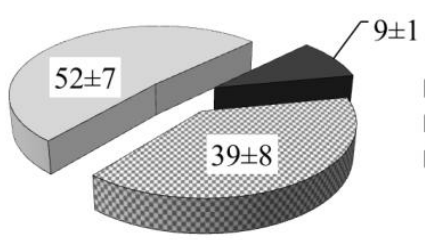

$\square \Sigma(3-29)$

- Low Angle, $<15^{\circ}$

圆 Random, High Angle

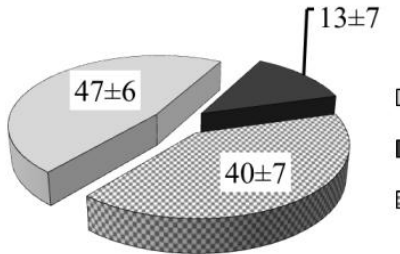

$\square \Sigma(3-29)$

$\square$ Low Angle, $<15^{\circ}$

圆 Random, High Angle

Figure 5. The grain boundary character distributions for the Inner sample (left) and the Outer sample (right).

The distribution of misorientation angles and CSL-related grain boundaries (shown in Figure 6a and Figure $6 \mathrm{~b}$, respectively) exhibit a high fraction of twin boundaries, $\sim 35 \%$, as indicated by the frequency at $\theta=60^{\circ}$ in Figure 6a, and the high fraction of $\Sigma 3$ boundaries in Figure 6b. Again, statistically, the distributions in misorientation and CSL-related boundaries are not significantly 
different between Inner and Outer samples. (The error bars in Fig. 6 were calculated by determining the distribution of each area analyzed for a given sample, Inner or Outer, determining the standard deviation for each abscissa value and then dividing the standard deviation by the square root of the number of regions analyzed, i.e. number of observations, to yield the standard error for each abscissa value.)

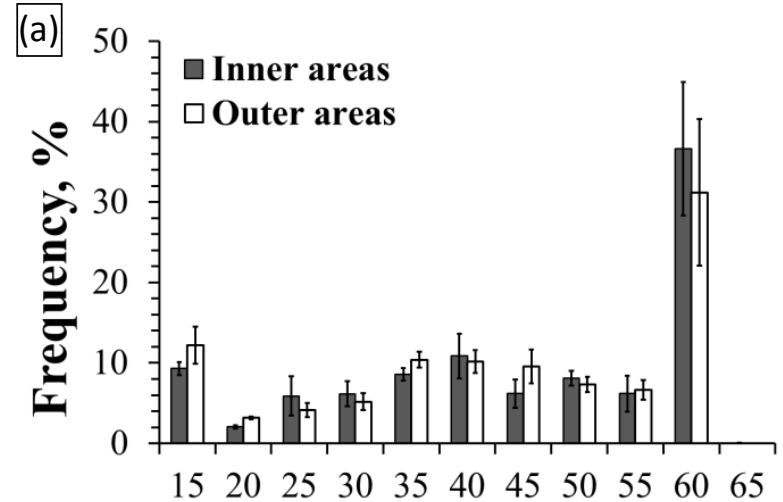

Misorientation Angle, ${ }^{\circ}$

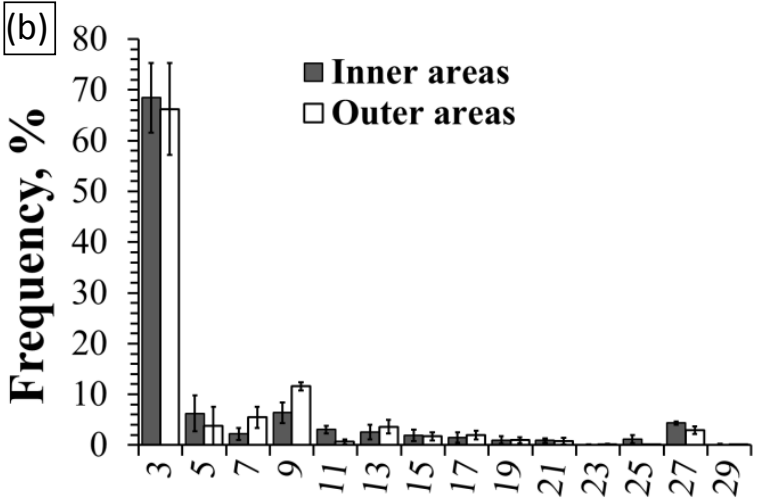

CSL-related Grain Boundaries, $\Sigma$

Figure 6. (a) Distribution of grain boundary misorientation angles and (b) the distribution of just CSLrelated grain boundaries (right).

\section{DISCUSSION}

The SiC layer of TRISO-coated fuel is designed as the primary containment for fission products in advanced high-temperature fuel. Therefore, the microstructure of the SiC layer has been of keen interest during the development of coated fuels, especially since there have been reports of the release of fission products through seemingly intact SiC layers [van der Merwe, 2009; Nabielek, et al., 1977; Demkowicz, et al., 2012]. As a result, there have been SEM-based EBSD studies on particles from the same composite batch (the composite batch consisted of TRISO particles fabricated in three separate production runs using the same nominal fabrication parameters, Hunn, et al, 2006) as the particle in Figure 1 [Kirchhofer, et al., 2013; Gerczak, 2013] and on particles containing a surrogate fuel kernel with a SiC layer deposited under similar conditions [Tan, et al., 2008]. These two studies were carried out on polished cross sections and interrogated the grain characteristics in the radial growth direction of the SiC layer, same as the FIB samples analyzed in this study. The main difference between the two cited studies and this work is the data acquisition step size, which was $50 \mathrm{~nm}$ for Kirchhofer et al., 2013 and $100 \mathrm{~nm}$ for Tan et al., 2008 versus the 10.4-nm step sized used in this study. In Tan et al.'s 2008 article, the TRISO-coated particle contained a surrogate fuel particle and the SiC layer was deposited at a slightly lower temperature of $1410^{\circ} \mathrm{C}$ compared to $1425^{\circ} \mathrm{C}$ for this study and the study in Kirchhofer et al.'s 2013 article. These differences are not expected to significantly affect the microstructure of the SiC layer. The fabrication conditions in the Kirchhofer study are nominally the same as those for the particle in this study, although small run-to-run variations may exist within the composite batch of particles. In the Tan et al. study, the slightly lower deposition temperature represents an almost negligible difference when the homologous temperature used in the fabrication of the SiC layer is considered, $T_{\text {Fab }}=0.560 T_{M P}$ for the Tan, et al., study versus $T_{F a b}=0.565 T_{M P}$ in this study. Therefore, the results presented here will be directly compared to these studies to evaluate the advantages and disadvantages of PED-based grain boundary analysis. 


\subsection{Grain Size}

This study found that the collection and analysis of grain orientation information from FIB-prepared TEM samples of the SiC layer of TRISO-coated fuel using precession diffraction techniques to be relatively easy and straightforward, yielding information with resolution on the nano-scale. However, for an accurate grain-size determination, the size of the scanned area must be considered. Figure 3 shows some relatively large grains are not fully enclosed by the scanned area, especially the orangecolored grain. Typically grains that intersect the edge of the scanned region are not included in the determination of grain size in the EDAX OIM software. As a result, the calculated average grain size is smaller than expected. This is evident when comparing the average grain size in this study with those in the other two studies, Table 1, in which the average grain size from SEM-based EBSD was found to be significantly greater $(>5 \times)$. As such, it is necessary in PED data acquisition to set up scan areas that are considerably larger than the largest grain in the sample.

Table 1. Summary of average grain size.

\begin{tabular}{|c|c|c|c|c|}
\hline & \multirow[b]{2}{*}{ Tan, et al., 2008} & \multirow{2}{*}{$\begin{array}{c}\text { Kirchhofer, et al., } \\
2013\end{array}$} & \multicolumn{2}{|c|}{ This Study } \\
\hline & & & Inner & Outer \\
\hline Data acquisition step size, $\mathrm{nm}$ & 100 & 50 & 10.4 & 10.4 \\
\hline Average Grain Size, nm & $800^{\mathrm{b}}$ & $<550^{\mathrm{a}}-750^{\mathrm{b}}$ & $131 \pm 135$ & $111 \pm 74$ \\
\hline
\end{tabular}

In the present study, the scan area was on the order of $2 \mu \mathrm{m} \times 2 \mu \mathrm{m}$, which was clearly still too small to capture the large grain in Figure 3. Scanning larger areas is possible, but this would result in a considerable increase in the data collection time. The scan time for a $2 \mu \mathrm{m} \times 2 \mu \mathrm{m}$ area in this study was on the order of 20-30 minutes. Doubling the size of the scan would result in a data collection time $4 \times$ greater-up to $2 \mathrm{hrs}$. At such long collection times sample drift may become an issue, although drift correction routines are available with the data collection software used in this study.

Finally, it should be mentioned that only the cubic form of SiC (3C) was assumed to be present in these samples. There is reference to a small volume fraction of the hexagonal form of $\mathrm{SiC}(6 \mathrm{H})$ present in SiC deposited by this method, albeit under slightly different conditions [Helary, et al., 2008]. However, no obvious stacking faults were observed, qualitatively indicating little, if any, SiC (6H) was present in these samples [Yang, et al., 2000; Yang, et al, 2001]. Others have not found or analyzed for SiC (6H) in SEM-based EBSD studies on CVD-deposited SiC [Helary,et al., 2006]. In the future, the PED data should also be evaluated for the presence of one or more hexagonal forms of $\mathrm{SiC}$, in addition to the cubic form, especially since this is relatively easy to do.

\subsection{Comparison of the Grain Character Distribution with Recent Literature}

The grain boundary character parameters are summarized in Table 2. The striking difference between the three studies lies in the fraction of low-angle grain boundaries. Both SEM-based EBSD studies report much higher fractions of low-angle grain boundaries compared to the present PEDbased study. (The EBSD study of Gerczak, 2013, reported a low angle grain boundary fraction similar to that reported here but the minimum misorientation angle considered in that work was $5^{\circ}$ whereas the minimum misorientation angle in this work and that of Kirchhofer was $2^{\circ}$. Therefore, the results of Gerczak will not be included in further discussions of the grain boundary character distribution.) 
Furthermore, there seems to be a direct correlation of the low-angle grain boundary fraction with data acquisition step size-the fraction of low-angle grain boundaries decreases with decreasing step size in Table 2. This is not unexpected since the number of dislocations between adjacent analysis points will increase with increasing distance between the adjacent analysis points for a uniform distribution of lattice dislocations. This is manifested as an increasing misorientation between adjacent analysis points as the step size increases since a small shift in orientation is associated with each individual lattice dislocation. In the data analysis software, a grain boundary is assumed to be present if the misorientation exceeds the threshold for the definition of a grain boundary (typically $2^{\circ}$ ). Therefore, a significant portion of the low-angle grain boundary fraction in SEM-based EBSD data may actually be due to a large-acquisition step size in a field of randomly distributed lattice dislocations rather than due to a dense tangle or organized set of lattice dislocations inherent to a true low-angle grain boundary. Alternatively, there is a remote possibility that sample preparation may play a role in the loss of dislocations to the free surface of the TEM sample. Generally, the preparation of electron transparent metallic alloys can result in the loss of screw dislocations to the foil surface due to image forces [Hirsch, et al., 1977]. However, no mention of significant loss of dislocations to the free surfaces of electron transparent TEM foils during FIB sample preparation of $\mathrm{SiC}$ has been reported in literature. Also, whether the mobility of dislocations in covalently bonded $\mathrm{SiC}$, due to the temperature rise or defect generation from ion bombardment during FIB thinning, is sufficient to cause dislocations to escape the TEM lamellae is speculative, at best. Conversely, mechanical grinding and polishing during the preparation of SEM samples may damage the surface under analysis during SEM-based EBSD, potentially yielding a high fraction of low-angle grain boundaries, especially when interrogated with a relatively large step size. Mechanical preparation damage of the SiC would be greatly reduced in the FIB-extracted TEM samples since the bulk of these samples come from below the mechanically prepared surface (the plane of the TEM samples are perpendicular to the polished surface of the metallographic mount). Additionally, most mechanical polishing damage may be removed during thinning of the TEM lamellae in the FIB, which is not observed during PED data collection. However, it should be noted that in the Kirchhofer, et al., 2013, study, FIB was used to "mill" the mechanically polished cross section prior to EBSD analysis and is expected to remove at least a majority, if not all, of the mechanical polishing damage. Finally, the differences in the low angle grain boundary fractions between this and the Kirchhofer, et al., 2013, study may arise from run-to-run variations where very small differences $(<3 \%)$ existed in the actual fabrication parameters for the three production runs used to generate the composite batch. Although both particles came from the same composite batch, it is not known whether they came from the same production run. Additional work is required to identify the cause(s) for the differences in low-angle grain boundary fraction between the PED-based and SEM-based SiC grain boundary data.

However, all three studies report a high fraction of high-angle grain boundaries. The study of Kirchhofer et al., 2013, reports CSL-related and high-angle grain boundary fractions similar to the present study, which is expected since the SiC layer analyzed in both studies was deposited with the same fabrication parameters. However, the study by Tan et al., 2008 reports a much lower CSLrelated grain boundary fraction. The cause of this discrepancy is not clear but may arise from the large step size used for data acquisition or possibly the slightly different fabrication conditions used for this TRISO-coated particle with a surrogate fuel kernel. Generally, the distributions of just the CSLrelated grain boundaries in Table 2 follow similar trends in all three studies with $\Sigma 3, \Sigma 9$, and $\Sigma 27$ representing the majority of CSL-related grain boundaries. 
Table 2. Comparison of grain boundary character parameters with literature.

\begin{tabular}{|c|c|c|c|c|}
\hline & \multirow{2}{*}{$\begin{array}{c}\text { Tan, et al., } \\
2008\end{array}$} & \multirow{2}{*}{$\begin{array}{l}\text { Kirchhofer, } \\
\text { et al., } 2013\end{array}$} & \multicolumn{2}{|c|}{ This Study } \\
\hline & & & Inner & Outer \\
\hline Data acquisition step size, $\mathrm{nm}$ & 100 & 50 & 10.4 & 10.4 \\
\hline Grain Boundary Character distribution & & & & \\
\hline Low angle $\left(2^{\circ} \leq\right.$ angle $\left.\leq 15^{\circ}\right)$ & 35.6 & 21 & $9 \pm 1.2$ & $13 \pm 5.1$ \\
\hline $\operatorname{CSL}(\Sigma 3-\Sigma 29)$ & 12.1 & 40 & $52 \pm 6.6$ & $47 \pm 6.2$ \\
\hline Random high angle $\left(>15^{\circ}\right.$ and not $\left.\Sigma 3-\Sigma 29\right)$ & 52.3 & 39 & $39 \pm 7.5$ & $40 \pm 5.1$ \\
\hline $\begin{array}{c}\text { CSL boundary distribution } \\
\text { (Percent of all CSL boundaries, } \Sigma 3-\Sigma 29)\end{array}$ & & & & \\
\hline$\Sigma 3$ & 59.5 & 67.5 & $68.4 \pm 6.8$ & $66.2 \pm 9.1$ \\
\hline$\Sigma 9$ & 20.7 & 12.5 & $6.4 \pm 2.0$ & $11.6 \pm 0.8$ \\
\hline$\Sigma 27$ & 12.4 & 2.5 & $4.4 \pm 0.3$ & $2.9 \pm 0.7$ \\
\hline Other $\Sigma$ & 7.4 & 17.5 & $20.9 \pm 8.2$ & $19.3 \pm 10.6$ \\
\hline
\end{tabular}

\subsection{Comments on TEM-based Orientation Mapping}

The obvious advantage of TEM-based PED orientation mapping is the improved spatial resolution. The electron beam/sample interaction volume is on the order of the electron beam area multiplied by the TEM sample thickness. Electron beam spreading in the TEM sample is minimal compared to SEM-based EBSD applications. However, the angular resolution is somewhat lower with PED compared to EBSD, which has claimed an angular resolution as low as $0.5^{\circ}$ [Prior, et al., 1999]. However, for general orientation mapping, high-angular resolution is not generally required.

As with SEM-based automated EBSD data acquisition, automated, point-by-point, orientation data acquisition in the TEM can require significant time. Smaller step sizes (therefore, higher spatial resolution) result in much longer data acquisition times for areas of the same size. Sample drift can be an issue, although this can be mitigated by using drift correction software. The alternative is to scan more areas of smaller dimensions to achieve the same statistical requirements, although this also adds to the total data collection time.

\section{CONCLUSIONS}

The quality of PED-derived orientation data was shown to improve with increasing sample thickness, although the quality of the data was considered to be acceptable regardless of the sample thickness used in this study ( 80-120 nm). Crystallographic orientation mapping and grain boundary analysis using TEM-based precession electron diffraction techniques result in much higher spatial resolution in FIB-prepared samples taken from the SiC layer of unirradiated TRISO-coated nuclear fuel than SEM-based EBSD studies found in literature. No statistical difference in the PED-derived grain boundary character and CSL-related grain boundary distributions were observed in areas of the SiC layer near the IPyC versus those areas near the OPyC layer. Analysis of grain boundary character from the TEM-based PED data showed a much lower fraction of low-angle grain boundaries compared to SEM-based EBSD data from the SiC layer of the same TRISO-coated particle, as well as a SiC layer deposited at a slightly lower temperature. It was concluded that the high fraction of low-angle grain boundaries found with EBSD likely resulted from a larger data acquisition step size compared to the TEM-based orientation data, surface damage during sample preparation, or loss of dislocations 
during FIB-preparation of TEM samples. However, grain boundary analysis of the data acquired via TEM-based PED resulted in fractions of high-angle and CSL-related grain boundaries similar to those found using SEM-based EBSD. Since the grain size of the SiC layer of TRSIO fuel can be as small as 250 $\mathrm{nm}$ [Kirchhofer, 2013], depending on the fabrication parameters, and grain boundary fission product precipitates in the SiC layer of irradiated TRISO particles can be nano-sized, the TEM-based PED orientation data collection method is preferred to determine an accurate representation of the relative fractions of low-angle, high-angle, and CSL-related grain boundaries. However, the TEMbased PED data collection times may be significantly longer so that the statistical requirements are met. Additionally, accurate characterization of the SiC grain size with TEM-based PED methods is possible, but significantly larger and more analysis areas than those used in this study are required.

\section{ACKNOWLEDGEMENTS}

This work was sponsored by the U.S. Department of Energy, Office of Nuclear Energy, under DOE Idaho Operations Office Contract DE-AC07-05ID14517, as part of the Very High Temperature Reactor Development Program as well as the National Science Users Facility Program. The authors would like to acknowledge the efforts of Mr. James Madden in the FIB-fabrication of the TEM samples as well as other staff at the Materials and Fuels Complex at INL and those at the Center for Advanced Energy Studies.

\section{REFERENCES}

Demkowicz, P., Hunn, J., Morris, R., Harp, J., Winston, P., Baldwin, C., Montgomery, F., 2012. Preliminary results of post-irradiation examination of the AGR-1TRISO fuel compacts, Paper HTR2012-3-021, Proc. Of the HTR 2012, Toykyo, Japan, October 28-November 1, 2012.

Gerczak, T., 2013, Understanding Ag Release from TRISO Fuel Through Surrogate Diffusion Experiments and Fuel Analysis, PhD. Dissertation, University of Wisconsin-Madison.

Helary, D., Dugne, O., Bourrat, X., Jouneau, P.H., Cellier, F., 2006. EBSD investigation of SiC for HTR fuel particles, J. Nuc. Matls., vol. 350, 332-335.

Helary, D., Dugne, O., Bourrat, X., 2008. Advanced characterization techniques for SiC and PyC coatings on high-temperature reactor fuel particles, J. Nuc. Matls., vol. 373, 150-156.

Hirsch, P., Howie, A., Nicholson, R., Pashley, D., Whelan, M., 1977. Electron Microscopy of Thin Crystals, Robert E. Krieger Publishing Co., Inc., Malabar, Florida.

Hunn, J.D. and Lowden, R.A., 2006. Data Compilation for AGR-1 Variant 3 Coated Particle Composite LEU01-49T, ORNL/TM-2006/022, May 2006

Khalil, S., Swaminathan, N., Schrader, D., Heim, A. J., Morgan, D.D., Szlufarska, I., 2011. Diffusion of Ag along $\Sigma 3$ grain boundaries in 3C-SiC, Physical Review B 84, 214104.

Kirchhofer, R., Hunn, J.D., Demkowicz, P.A., Cole, J.I. and Gorman, B.P., 2013. Microstructure of TRISO coated particles from the AGR-1 experiment: SiC grain size and grain boundary character, J. Nuc. Matls., vol. 432, 127-134.

Lillo, T.M. and van Rooyen, I.J., 2015, 2015. Associations of Pd, $\mathrm{U}$ and Ag in the SiC layer of neutronirradiated TRISO fuel, J. Nuc. Matls, 460, 97-106. 
Maki, J.T., 2009. AGR-1 Irradiation Experiment Test Plan, INL.EXT-05-00593, rev. 3.

Malherbe, J.B., 2013. Diffusion of fission products and radiation damage in SiC, J. Phys. D: Appl. Phys. 46 (2013) 473001, doi:10.1088/0022-3727/46/47/473001

Nabielek, H., Brown, P.E., Offerman, P., 1977. Silver Release from Coated Particle Fuel, Nucl. Techn., 35, 483-493.

Prior, D. J., Boyle, A. P., Brenker, F., Cheadle, M. C., Day, A., Lopez, G., Peruzzo, L., Potts, G. J., Reddy, S., Spiess, R., Timms, N. E., Trimbly, P., Wheeler, J., Zetterström, L., 1999. The application of electron backscatter diffraction and orientation contrast imaging in the SEM to textural problems in rocks, Amer. Miner., 84, 1741-1759.

Rabone, J. and López-Honorato, E., 2015. Density functional theory metadynamics of silver, caesium and palladium diffusion at $\beta-S i C$ grain boundaries, J. Nuc. Matls., 458, 56-63.

Rauch, E.F., Véron, M., Portillo, J., Bultreys,D., Maniette, Y. and Nicolopoulos, S., 2008. Automatic Crystal Orientation and Phase Mapping in TEM by Precession Diffraction, Micro. and Anal., Nanotechnolgy Supplement, Nov. 2008 S5-S8.

Schrader, D., Khalil, S. M., Gerzcak, T., Allen, T.R., Heim, A. J., Szlufarska, I., Morgan, D., 2011. Ag diffusion in cubic silicon carbide, J. Nucl Mater, 408, 257-271.

Tan, L., Allen, T.R., Hunn, J.D., Miller, J.H., 2008. EBSD for microstructure and property characterization of the SiC-coating in TRISO fuel particles, J. Nuc. Matls., vol. 372, 400-404.

van der Merwe, J. J., 2009. Evaluation of silver transport through SiC during the German HTR fuel program, J. Nucl. Mater., 395, 99-111.

van Rooyen, I.J., Lillo, T.M., Wu, Y.Q., 2014. Identification of silver and palladium in irradiated TRISO coated particles of the AGR-1 experiment, J. Nucl. Mater., 446, 178-186.

Yang, X., Wu, Y., Ye, H., 2000. The $\alpha \rightarrow \beta$ polytypic transformation in SiC induced by ball milling, Philo. Mag. Letters, vol. 80, 333-339.

Yang, X., Wu, Y., Ye, H., 2001. High-resolution electron microscopy observations of microplastic fracture in SiC under ball milling at room temperature, Philo. Mag. Letters, vol. 81, 1-8. 
Demkowicz, P., Hunn, J., Morris, R., Harp, J., Winston, P., Baldwin, C., Montgomery, F., 2012. Preliminary results of post-irradiation examination of the AGR-1TRISO fuel compacts, Paper HTR2012-3-021, Proc. Of the HTR 2012, Toykyo, Japan, October 28-November 1, 2012.

Gerczak, T., 2013, Understanding Ag Release from TRISO Fuel Through Surrogate Diffusion Experiments and Fuel Analysis, PhD. Dissertation, University of Wisconsin-Madison.

Helary, D., Dugne, O., Bourrat, X., Jouneau, P.H., Cellier, F., 2006. EBSD investigation of SiC for HTR fuel particles, J. Nuc. Matls., vol. 350, 332-335.

Helary, D., Dugne, O., Bourrat, X., 2008. Advanced characterization techniques for SiC and PyC coatings on high-temperature reactor fuel particles, J. Nuc. Matls., vol. 373, 150-156.

Hirsch, P., Howie, A., Nicholson, R., Pashley, D., Whelan, M., 1977. Electron Microscopy of Thin Crystals, Robert E. Krieger Publishing Co., Inc., Malabar, Florida.

Hunn, J.D. and Lowden, R.A., 2006. Data Compilation for AGR-1 Variant 3 Coated Particle Composite LEU01-49T, ORNL/TM-2006/022, May 2006

Khalil, S., Swaminathan, N., Schrader, D., Heim, A. J., Morgan, D.D., Szlufarska, I., 2011. Diffusion of Ag along $\Sigma 3$ grain boundaries in 3C-SiC, Physical Review B 84, 214104.

Kirchhofer, R., Hunn, J.D., Demkowicz, P.A., Cole, J.I. and Gorman, B.P., 2013. Microstructure of TRISO coated particles from the AGR-1 experiment: SiC grain size and grain boundary character, J. Nuc. Matls., vol. 432, 127-134.

Lillo, T.M. and van Rooyen, I.J., 2015, 2015. Associations of Pd, $\mathrm{U}$ and $\mathrm{Ag}$ in the SiC layer of neutronirradiated TRISO fuel, J. Nuc. Matls, 460, 97-106.

Maki, J.T., 2009. AGR-1 Irradiation Experiment Test Plan, INL.EXT-05-00593, rev. 3.

Malherbe, J.B., 2013. Diffusion of fission products and radiation damage in SiC, J. Phys. D: Appl. Phys. 46 (2013) 473001, doi:10.1088/0022-3727/46/47/473001

Nabielek, H., Brown, P.E., Offerman, P., 1977. Silver Release from Coated Particle Fuel, Nucl. Techn., 35, 483-493.

Prior, D. J., Boyle, A. P., Brenker, F., Cheadle, M. C., Day, A., Lopez, G., Peruzzo, L., Potts, G. J., Reddy, S., Spiess, R., Timms, N. E., Trimbly, P., Wheeler, J., Zetterström, L., 1999. The application of electron backscatter diffraction and orientation contrast imaging in the SEM to textural problems in rocks, Amer. Miner., 84, 1741-1759.

Rabone, J. and López-Honorato, E., 2015. Density functional theory metadynamics of silver, caesium and palladium diffusion at $\beta$-SiC grain boundaries, J. Nuc. Matls., 458, 56-63. 
Rauch, E.F., Véron, M., Portillo, J., Bultreys,D., Maniette, Y. and Nicolopoulos, S., 2008. Automatic Crystal Orientation and Phase Mapping in TEM by Precession Diffraction, Micro. and Anal., Nanotechnolgy Supplement, Nov. 2008 S5-S8.

Schrader, D., Khalil, S. M., Gerzcak, T., Allen, T.R., Heim, A. J., Szlufarska, I., Morgan, D., 2011. Ag diffusion in cubic silicon carbide, J. Nucl Mater, 408, 257-271.

Tan, L., Allen, T.R., Hunn, J.D., Miller, J.H., 2008. EBSD for microstructure and property characterization of the SiC-coating in TRISO fuel particles, J. Nuc. Matls., vol. 372, 400-404.

van der Merwe, J. J., 2009. Evaluation of silver transport through SiC during the German HTR fuel program, J. Nucl. Mater., 395, 99-111.

van Rooyen, I.J., Lillo, T.M., Wu, Y.Q., 2014. Identification of silver and palladium in irradiated TRISO coated particles of the AGR-1 experiment, J. Nucl. Mater., 446, 178-186.

Yang, X., Wu, Y., Ye, H., 2000. The $\alpha \rightarrow \beta$ polytypic transformation in SiC induced by ball milling, Philo. Mag. Letters, vol. 80, 333-339.

Yang, X., Wu, Y., Ye, H., 2001. High-resolution electron microscopy observations of microplastic fracture in SiC under ball milling at room temperature, Philo. Mag. Letters, vol. 81, 1-8. 